$\mathrm{RM}-76-42$

\title{
ILLUSION OR REALITY?: \\ THE MATHEMATICS OF ATTAINABLE GOALS \\ AND IRREDUCIBLE UNCERTAINTIES
}

J. Casti

May 1976

Research Memoranda are interim reports on research being conducted by the International Institute for Applied Systems Analysis, and as such receive only limited scientific review. Views or opinions contained herein do not necessarily represent those of the Institute or of the National Member Organizations supporting the Institute. 


\section{Preface}

A substantial number of IIASA projects involve questions of how to attain certain goals within constraints on admissible policies and/or how much information about a given process is obtainable from measurements made upon only parts of the system. This report examines these questions from the viewpoint of mathematical system theory and illustrates the methodological apparatus by examples taken from previous IIASA work in ecology, urban, water, and energy. 


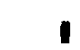




\author{
Illusion or Reality?: \\ The Mathematics of Attainable Goals \\ and Irreducible Uncertainties
}

J. Casti

\begin{abstract}
In complex, high-dimensional systems, it is usually far from obvious what states are attainable within the constraints on admissible actions. Similarly, when only parts of the system are physically measurable, a vital practical as well as philosophical question arises as to how much inherent uncertainty remains in determining the true state of the system. In system-theoretic jargon, these are problems of reachability and constructibility.
\end{abstract}

This paper presents an overview of the current mathematical state-of-the-art as it relates to the reachability/constructibility question. Particular emphasis is given to those results which seem most useful for dealing with practical system problems. After an introductory section to broadly motivate the subject, a survey of the principal mathematical results for linear, bilinear, and multilinear systems is given, along with a discussion of multidimensional nonlinear problems. The paper concludes with several actual problems from ecology, urban systems, water resource systems, transportation networks, and energy systems where reachability and/or constructibility questions play an essential role in the analysis.

I. Introduction

A few of the many pitfalls facing the international air traveller in these days of ever-changing schedules, flight cancellations, hijackings, and weather diversions are the words "you can't get there from here," uttered by ticket sellers and travel agents and/or "where are we now," coming from weary fellow travellers. These are particularly homey examples of the general system-theoretic problems of reach- 
ability and constructibility: a system (a set of cities) is given, along with a set of admissible inputs (schedules) and means of observation (visual recognition). The problem of reachability is to characterize all those states (cities) "reachable" by application of admissible inputs. The problem of constructibility is to determine all those states which may be uniquely identified from knowledge of past outputs (observations) and the initial state. Clearly, the airline situation presents us with a system which is neither completely reachable nor completely constructible since, by the opening remarks, there are cities which are unreachable (those without airline service) and unconstructible (those not visually distinguishable or those which represent unscheduled stops).

The underlying question to be posed here is: How can mathematics contribute to systems analysis? "Mathematics" will mean here exactly the same thing as it does to the contemporary professional mathematician, namely theorems, methods of proof, natural constructions, and so forth. In this paper, we will not examine questions involving data analysis, statistics, numerical formulas, and other pedestrian (though often very useful) things which to the layman are also "mathematics."

We pose the foregoing question because of our optimistic prejudice that mathematics is significant in systems analysis and research. The only rational argument supporting such a position is via analogy. Each developing and "hardening" science (chemistry, physics, economics, psychology,...) gradually reaches a stage beyond which progress is impossible without organized knowledge, because the implications of existing knowledge are too complex to digest without the help of abstraction, that is, without mathematics. In physics, this situation occurred well before the end of the $19^{\text {th }}$ century. We do not wish to start dreaming in print as to how and in what form this evolutionary stage will finally emerge in systems analysis. We shall merely try to outline what 
has and might be expected to happen within the narrow confines of determining attainable goals and ascertaining the limitations of information from measurement as these problems pertain to modern systems.

A moment's thought is sufficient to be convinced that the properties of reachability and constructibility are fundamental system concepts in that they delineate what is and is not possible within given structure and interaction constraints. If one accepts the view that good systems analysis, like good politics, is the art of the possible, then it is difficult to escape the conclusion that a thorough investigation of the reachable and constructible states should be carried out at a very early stage in the analysis of any model proposed to represent a given system. There is no utility in striving to achieve theoretically unobtainable goals or in attempting to resolve fundamentally indistinguishable situations (shades of quantum mechanics and the uncertainty principle).

It is our intention in this paper to briefly survey the current state of the art in the mathematical system theory world as it pertains to the reachability/constructibility issue. We shall strive to present the results in as intuitive a manner as possible, preferring in many places to replace absolutely precise statements of various results by weakened, but more transparent, versions conveying the essence of the original result. In this connection, we shall omit all proofs of theorems concentrating our attention instead upon the meaning and relevance of the results to problems of applied systems analysis. However, before setting sail upon the high seas of analysis and algebra, we consider some elementary examples to more completely convey the scope and meaning of the basic problems involved in this study.

\section{A. Electrical Circuits}

Consider the RLC electrical network shown in Figure 1. 


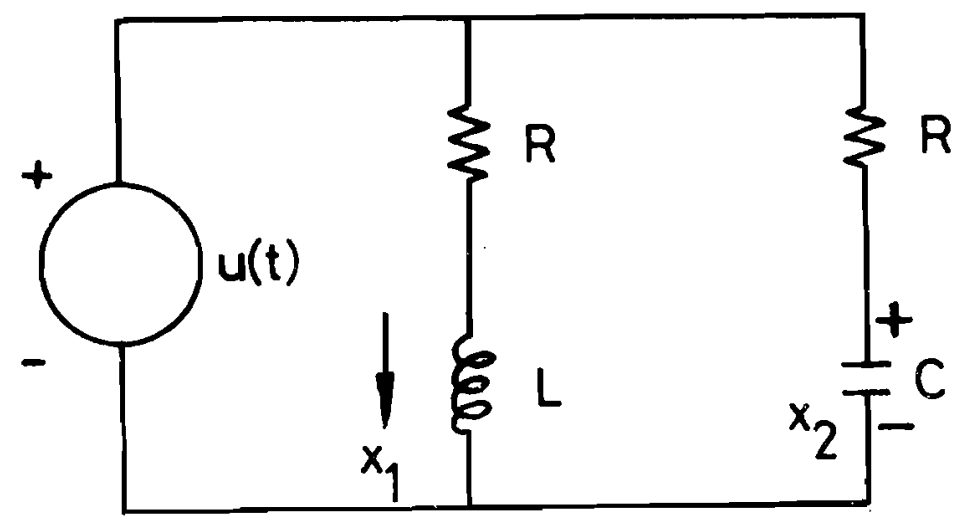

Figure 1. RLC Circuit

The magnetic flux at time $t$ is denoted by $x_{1}(t)$, while $x_{2}(t)$ is the electric charge on the capacitor. The input $u(t)$ is a voltage source. If $L / C=R^{2}=1$, the dynamical equations for this system are

$$
\begin{aligned}
& \frac{d x_{1}}{d t}=-\frac{1}{L} x_{1}(t)+u(t), \\
& \frac{d x_{2}}{d t}=-\frac{1}{C} x_{2}(t)+u(t)
\end{aligned}
$$

If we let

$$
\bar{x}_{1}=x_{1}+x_{2}, \quad \bar{x}_{2}=x_{1}-x_{2},
$$

it is easily verified that 


$$
\begin{aligned}
& \frac{d \bar{x}_{1}}{d t}=-\frac{1}{L} \bar{x}_{1}(t)+2 u(t), \\
& \frac{d \bar{x}_{2}}{d t}=-\frac{1}{L} \bar{x}_{2}(t),
\end{aligned}
$$

Thus, the input voltage affects only the state $\bar{x}_{1}$, while $\bar{x}_{2}$ cannot be influenced by the applied voltage source. In terms of the original variables, if the system begins in the equilibrium state $x_{1}(0)=x_{2}(0)=0$, the only states which may be reached by application of the input voltage $u(t)$ are those where $x_{1}(t)=x_{2}(t), i . e .$, those in which the electric charge and magnetic flux are equal.

Now assume that the measuring apparatus is such that we have only the capability of measuring the variable $\bar{x}_{2}(t)$, i.e., the system output is $\bar{y}(t)=\bar{x}_{2}(t)$. Then it is clear that knowledge of the past outputs and the initial system state will yield no information whatsoever about the state $\bar{x}_{1}$. Thus, only states of the form $\bar{x}_{1}=0, \bar{x}_{2}=\bar{x}_{2}$ may be determined from the output $\bar{y}$ or, in the original variables, only states for which the magnetic flux equals the negative of the electric charge.

\section{B. Macroeconomics [1]}

A highly simplified version of a common economic situation will illustrate another side of the reachability issue. A country has the short-run economic objective of full employment without inflation (internal balance) and balance of international payments (external balance), which must be accomplished through policy instruments such as changes in the interest rate and in the budget deficit. Thus, the country has a central bank which controls interest rates, and a legislative body (congress) which controls changes in the government deficit. While it is politically difficult to combine these institutions into a single controlling agency, it is possible to establish general directives for them to follow. The question is 
whether or not this system can be controlled. To study this problem, we define the following variables

$$
\begin{aligned}
& \mathrm{Y}(\mathrm{t})=\text { domestic production (= income of consumers) } \\
& \mathrm{X}(\mathrm{t})=\text { aggregate expenditures } \\
& \mathrm{C}(\mathrm{t})=\text { aggregate consumption } \\
& \mathrm{S}(\mathrm{t})=\text { aggregate savings } \\
& \mathrm{I}(\mathrm{t})=\text { domestic investment } \\
& \mathrm{M}(\mathrm{t})=\text { imports of foreign goods and services } \\
& \mathrm{K}(\mathrm{t})=\text { net capital outflow } \\
& \mathrm{T}(\mathrm{t})=\text { net taxes of transfers } \\
& \mathrm{G}(\mathrm{t})=\text { government expenditures for goods and services } \\
& \mathrm{B}(\mathrm{t})=\text { net surplus in international balance of payments }
\end{aligned}
$$

All of the above variables are annual rates in period $t$, and are deflated to a uniform price level. Define the additional variables

$$
\begin{aligned}
& E= \text { exports of goods and services (assumed constant) } \\
& Y_{F}= \text { the full employment, no inflation level of domestic } \\
& \text { production, (assumed constant) } \\
& r(t)= \text { domestic interest rate } \\
& r_{F}= \text { foreign interest rate (assumed constant) } \\
& \text { Four accounting identities link these variables: } \\
& \qquad=C+S+T, \\
& X=C+I+K+G, \\
& B=E-M-K \\
& B=
\end{aligned}
$$

Further, the following linear relations have been found empirically to be roughly valid: 


$$
\begin{aligned}
& S=\alpha_{1} Y-\alpha_{0}, \\
& M=\beta_{1} Y-\beta_{0}, \\
& I=\gamma_{1} r+\gamma_{0}, \\
& K=-\delta_{1} r+r_{0},
\end{aligned}
$$

Defining the state and control vectors

$$
x(t)=\left(\begin{array}{c}
B(t) \\
Y(t)
\end{array}\right), \quad u(t)=\left(\begin{array}{l}
r(t+1)-r(t) \\
D(t+1)-D(t)
\end{array}\right),
$$

where $\mathrm{D}=\mathrm{G}-\mathrm{T}$ (net government deficit), the above relations give the dynamical model

$$
x(t+1)=x(t)+A u(t),
$$

where

$$
A=\left[\begin{array}{cc}
\delta_{1}+\mu \beta_{1} \gamma_{1} & -\mu \beta_{1} \\
-\mu \gamma_{1} & \mu
\end{array}\right], \quad\left(\mu=1 /\left(\alpha_{1}+\beta_{1}\right)\right)
$$

From a given initial state $\mathrm{x}(0)$, the planner desires to steer the economy to the target

$$
x^{*}=\left[\begin{array}{l}
0 \\
Y_{F}
\end{array}\right],
$$

where the international payments are in balance and a level $Y_{F}$ of outputs is achieved yielding internal balance.

For the above example, it doesn't take any deep reasoning to see that with unrestricted changes in tax rate and deficits, any desired terminal state can be reached if the 
matrix $A$ is nonsingular. Since this will be the case for almost every set of values of the paramters $\delta_{1}, \beta_{1}, \gamma_{1}, \alpha_{1}$, the complete reachability property is "stable" with respect to changes in these parameters.

Now assume the more realistic situation in which the controlling influences are dictated on the basis of measurements of $x(t)$, i.e., every admissible control has the timeinvariant linear feedback form

$$
\mathrm{u}(\mathrm{t})=\mathrm{H}\left(\mathrm{x}-\mathrm{x}^{*}\right),
$$

where $\mathrm{H}$ is a $2 \times 2$ matrix. With decentralization of control responsibility and limited information on the state of the system, we are interested in the behavior of the system

$$
x(t+1)=x(t)+A H\left(x-x^{*}\right)
$$

The most central question is one of stability: does there exist any control policy such that $x(t) \rightarrow x^{*}$ as $t \rightarrow \infty$ ? In this connection, consider the case in which the central bank observes the balance of payments, the congress observes the output level, and no communication between the two takes place. Then $\mathrm{H}$ must be a diagonal matrix for each admissible control. For example, the set of admissible inputs might be

$$
\mathscr{H}=\left\{\mathrm{H}: \mathrm{H}_{1}=\left[\begin{array}{cc}
\mathrm{h}_{1} & 0 \\
0 & \mathrm{~h}_{2}
\end{array}\right], \quad\left|\mathrm{h}_{\mathrm{i}}\right| \leq 1\right\} .
$$

Direct calculation of the characteristic roots of the system (1) for $\mathrm{H} \varepsilon \mathscr{H}$ and arbitrary $A$ shows that this system is reachable and asymptotically stable if and only if $A$ is nonsingular and $A$ has at least one nonzero diagonal element. 


\section{Nonlinear Springs}

It seems reasonable to conjecture that the geometric shape of the reachable set should be a convex region, i.e., if $\mathrm{x}$ and $y$ are two points which are reachable, then any point on the line joining $x$ and $y$ should be reachable. For linear systems this is true; however, to see just how far astray one's intuition may be led, we present a simple example illustrating that not only is the reachable set not always convex, but it may not even be simply-connected.

Consider the harmonic oscillator with a "controllable" spring. It is described by the bilinear system

$$
\begin{array}{ll}
\dot{x}_{1}(t)=x_{2}(t), & x_{1}(0)=1, \\
\dot{x}_{2}(t)=-x_{1}(t)+u(t) x_{1}(t), & x_{2}(0)=0,
\end{array}
$$

where $x_{1}$ is the displacement and $x_{2}$ the velocity of the spring. For $|u(t)| \leq \varepsilon \ll 1$, the reachable set at $t$ ime $T$ for $T$ small is topologically equivalent to a disc but, for T sufficiently large, the reachable set encircles the origin (see Figure 2).

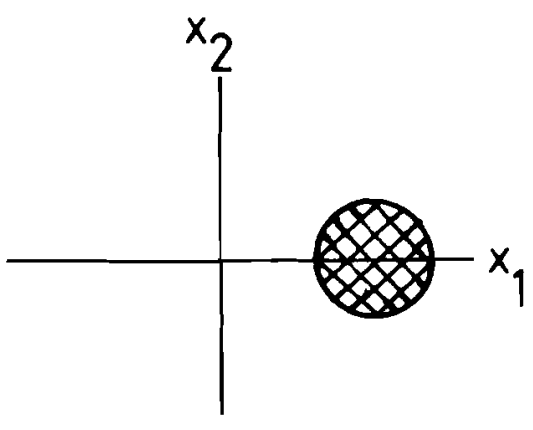

(a) $\operatorname{small} \mathrm{T}$

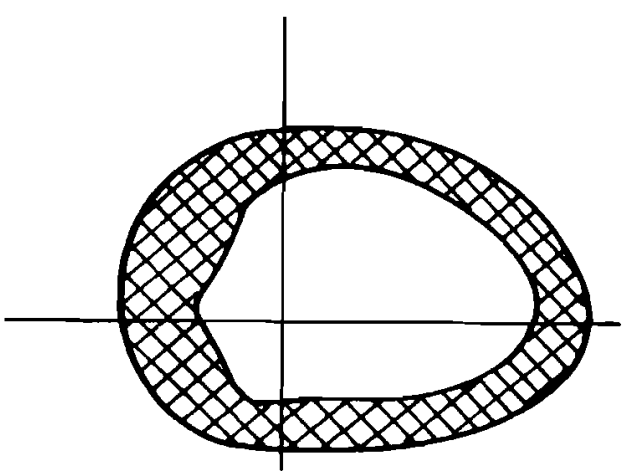

(b) large $\mathrm{T}$

Figure 2. The Reachable set for the "Controllable" Spring

The difficulty in this example is that the free motion of the system is an undamped oscillation. If we are to establish that the reachable set is equivalent to an n-ball, then we must adopt hypotheses which will exclude this "disconnected" type of behavior. If we ask that the reachable set be convex, 
the assumptions will have to be still stronger. For example, for the system

$$
\dot{\mathrm{x}}=\mathrm{u}(\mathrm{t}) \mathrm{Bx}(\mathrm{t}), \mathrm{x}(0)=\mathrm{x}_{0},
$$

the reachable set is the image of the admissible controls under the inap

$$
x(t)=\exp \int_{0}^{t} u(s) d s B x_{0} .
$$

This set will not be convex for $-1 \leq \mathrm{u} \leq 1$, say, unless $\mathrm{B}^{2}=\lambda \mathrm{B}$. Convexity in this case follows from the expression

$$
\exp B \alpha=I+f(\alpha) B
$$

In the absence of such a condition, there exist vectors $\mathrm{x}_{0}$ such that (exp $B \alpha) x_{0}$ is not convex for $-1 \leq \alpha \leq 1$.

For those readers whose primary interest is application, the following section on methodology may be skipped over with little loss in continuity. However, we recommend that the material be, at least, briefly skimmed as it will make the application section more meaningful by clarifying what one can reasonably expect in the way of methodological help utilizing the tools of mathematical system theory.

\section{Survey of Methodology}

In a brief paper such as this, it is clearly impossible to do justice to the vast literature on reachability/constructibility and to present even a small fraction of the useful and important results. Thus, what follows represents a somewhat electric selection of basic results which are either particularly simple, useful, and/or indispensable for dealing with the basic issues involved. We separate the class of systems into three groups: linear, bilinear (or multilinear), and nonlinear. As one might suspect, the only case which may be considered to be under more or less complete control is the 
linear situation, although substantial results are available also for the bilinear case. For these reasons, we have felt it desirable to progress from the state of rather complete knowledge to almost total ignorance in our survey of the current state of this branch of mathematical system theory.

For the purpose of basic definitions, we consider the system $\Sigma$ described by the equations

$$
\sum: \quad \dot{x}=f(x, u, t), \quad x\left(t_{0}\right)=x_{0},
$$

For simplicity, we assume that $f(0,0, t)=0, h(0, t)=0$, for all $t \geq t_{0}$, and that $f$ and $h$ are continuous functions of their arguments. We let $\phi(t ; \tau, x, u)$ denote the solution of (2) corresponding to an initial state $x$ at time $\tau$ under application of the input function $u(s), \tau \leq s \leq t$.

Definition 1. An event $\left(\tau, x^{*}\right)$ is controllable if and only if there exists a $\bar{t}>t_{0}$, and an admissible input $u \varepsilon \Omega$, (both $\bar{t}$ and $u$ may depend on $\left(\tau, x^{*}\right)$ ) such that

$$
\phi\left(\bar{t} ; \tau, x^{*}, u\right)=0
$$

$\Sigma$ is completely controllable if it is controllable for every event $\left(\tau, x^{*}\right)$.

In other words, an event is controllable if and only if it can be transferred to the origin in finite time by application of some admissible input function $u$.

Definition 2. An event $\left(\tau, x^{*}\right)$ is reachable if and only if there is an $\bar{s}>0$, and $a u \varepsilon \Omega$ (both $\bar{s}$ and $u$ may depend upon $\left.\left(\tau, x^{*}\right)\right)$, such that

$$
x^{*}=\phi(\tau ; \bar{s}, 0, u)
$$

$\Sigma$ is completely reachable if and only if it reachable for every event $\left(\tau, x^{*}\right)$. 
Graphically, controllability and reachability are as in Figure 3 .

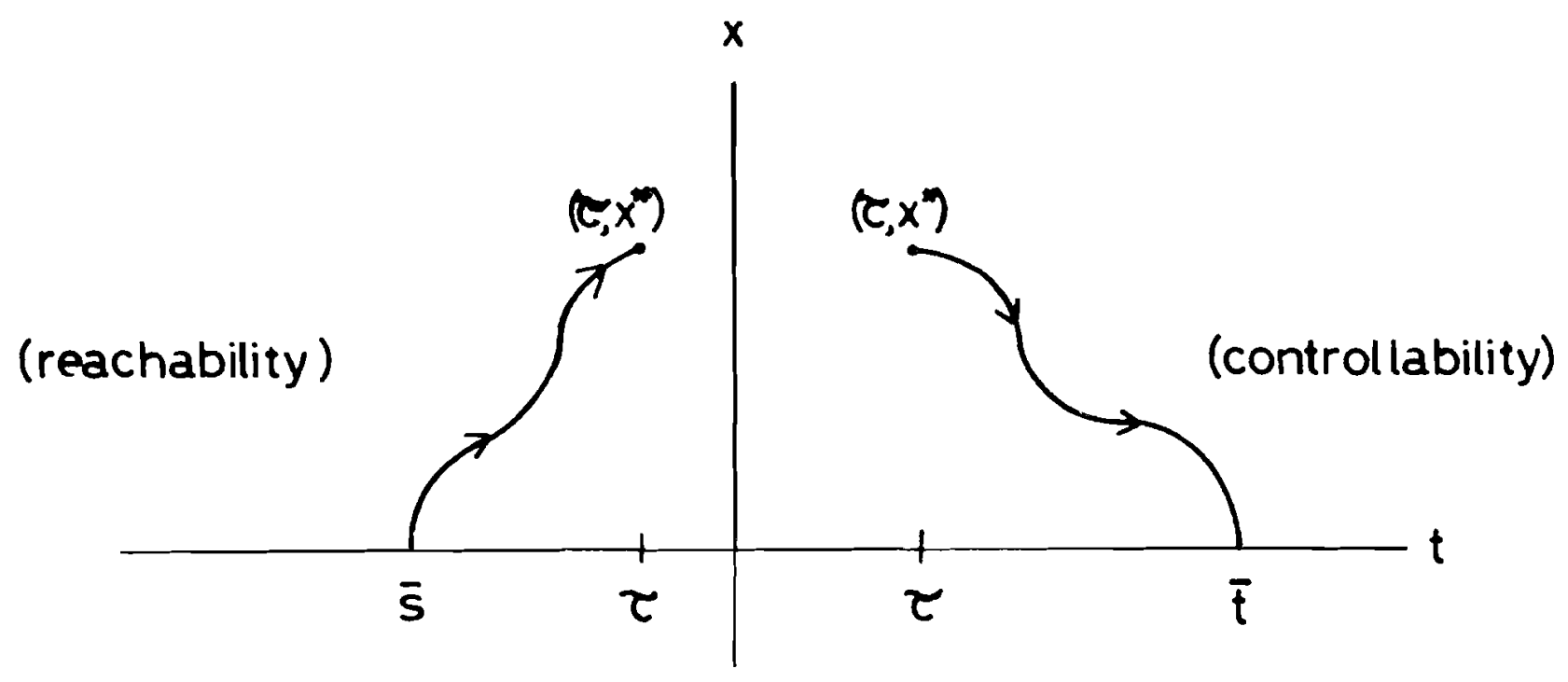

Figure 3. Controllability and Reachability

It is important to rote that controllability and reachability are entirely different concepts. They coincide only in special cases, one of which is when $\Sigma$ is a constant, continuous time, linear system. Caution! Even if a linear system is completely reachable and completely controllable, it does not follow that any event $(\tau, x)$ may be transferred to any other event $\left(\tau_{1}, x_{1}\right), \tau_{1} \geq \tau$, by suitable choice of input.

The notions dual to controllability/reachability are those of constructibility and observability. Basic definitions are:

Definition 3 . Two events $\left(\tau, x_{1}\right),\left(\tau, x_{2}\right)$ of a dynamical system belong to the same observation class (are indistinguishable in the future) if and only if

$$
h\left(\phi\left(t ; \tau, x_{1}, u\right), t\right)=h\left(\phi\left(t ; \tau, x_{2}, u\right), t\right)
$$


for all $t \geq \tau$ and all inputs $u(t), t \geq \tau$. (Note: $\phi(t ; \tau, \bar{x}, u) \triangleq x(t)$. ) The complementary notion is given by

Definition 4. Two events $\left(\tau, x_{1}\right),\left(\tau, x_{2}\right)$ of a dynamical system belong to the same reconstruction class (are indistinguishable in the past) if and only if

$$
h\left(\phi\left(\sigma ; \tau, x_{1}, u\right), \sigma\right)=h\left(\phi\left(\sigma ; \tau, x_{2}, u\right), \sigma\right)
$$

for all $\sigma \leq \tau$ and all $u(t), \tau \leq t \leq \sigma$.

These definitions are needlessly elaborate in the linear case. It is easily verified that if $f$ and $h$ are linear, we can re-phrase Definitions 3 and 4 as

Definitions $3^{\prime}$. An event $(\tau, x)$ of a linear dynamical system is unobservable if and only if it belongs to the observation class of $(\tau, 0)$, i.e., if and only if

$$
h(\phi(t ; \tau, x, 0), t)=0
$$

for all $t \geq \tau$.

Definition 4'. An event $(\tau, x)$ of a linear dynamical system is unconstructible if and only if it belongs to the reconstruction class of $(\tau, 0)$, i.e., if and only if

$$
\mathrm{h}(\phi(\sigma ; \tau, \mathrm{x}, 0), \sigma)=0,
$$

for all $\sigma \leq \tau$.

The motivation for Definition $3^{\prime}$ is clear: the "occurrence" of an unobservable event cannot be detected by looking at the output of the system after time $\tau$. Definition 4' says that the current state of a system at time $\tau$ cannot be determined by its past output up to time $\tau$ if that state is unconstructible.

A. Linear Systems

We consider the constant, linear version of $\Sigma$ in which

$$
\begin{aligned}
f(x, u, t) & =F x+G u, \quad x(0)=0, \\
h(x, t) & =H x,
\end{aligned}
$$


where $F, G, H$ are constant $n \times n, n \times m, p \times n$ matrices, respectively. In this case, the notions of reachability and controllability coincide, as do observability and constructibility. Due to the pioneering efforts of kalman and others, we have at our disposal very detailed descriptions of the the sets of reachable and constructible states. The single most important result in this direction is the following re-statement of a result first presented in [4].

Theorem 1. Define the $\mathrm{n} \times \mathrm{nm}$ controllability matrix $\mathscr{B}$ as

$$
\mathscr{B}=\left[G|F G| \ldots \mid F^{n-1} G\right] .
$$

Let $\Omega$ be the set of piecewise continuous functions on $[0, \infty]$. Further, let $\mathscr{L}=\left\{\mathrm{c}^{(1)}, \mathrm{c}^{(2)}, \ldots, \mathrm{c}^{(\mathrm{r})}\right\}$ be a maximal linearly independent set of vectors from the columns of $\mathscr{C}$. Then the set $\Re$ of reachable (or controllable) states of $\sum$ coincides with the subspace of $\mathrm{R}^{\mathrm{n}}$ spanned by the set $\mathscr{f}$.

Remarks :

(1) The fact that the reachable set is a subspace insures that it is convex.

(2) In general, the set $\mathscr{S}$ is not unique. All that is needed to characterize the reachable set is any maximal linearly independent subset from the columns of

(3) Given a particular $\left(\tau, x^{*}\right)$ which lies in $\mathscr{L}$, Theorem 1 gives no information as to what control $u(t) \varepsilon \Omega$ would lead from the origin to $x^{*}$. In general, one would have to solve the following Fredhom integral equation of the first kind for such an input

$$
x^{*}(\tau)=\int_{0}^{\tau} e^{F(\tau-s)} \mathrm{Gu}(s) d s .
$$

An alternate prescription involving generalized inverses is described in [19].

(4) Theorem 1 remains partially valid if the continuous system (4) is replaced by the discrete-time system

$$
\mathrm{x}_{\mathrm{k}+1}=\mathrm{Fx}_{\mathrm{k}}+\mathrm{Gu}_{\mathrm{k}}
$$


The reachable set is still given by the set $\mathscr{L}$, however, the controllable set is given by $\mathscr{L}$ only if the additional condition $\operatorname{det} F \neq 0$ is imposed.

Some of the useful corollaries of Theorem 1 are

Corollary 1. A constant system $\Sigma=(F, G,-)$ is completely reachable if and only if there is no nontrivial characteristic vector of $F$ which is orthogonal to every column of $G$.

Corollary 2. A constant system $\Sigma=(F, G,-)$ is completely reachable if and only if the smallest $F$-invariant subspace of $\mathrm{R}^{\mathrm{n}}$ containing the columns of $\mathrm{G}$ is $\mathrm{R}^{\mathrm{n}}$ itself.

Corollary 3. The state space of $\sum$ may be decomposed into the direct sum

$$
\mathrm{R}^{\mathrm{n}}=\mathrm{x}_{1} \oplus \mathrm{x}_{2}
$$

which decomposes the system dynamics as

$$
\begin{aligned}
& \frac{d x_{1}}{d t}=F_{11} x_{1}+F_{12} x_{2}+G_{1} u(t), \\
& \frac{d x_{2}}{d t}=F_{22} x_{2} .
\end{aligned}
$$

The subsystem $\left(F_{11}, G_{1},-\right)$ is completely reachable.

\section{Remark:}

In a problem in which control enters, only the space $x_{1}$ has meaning. Thus, it is essential to isolate the space $x_{1}$ at the outset as it may be of much lower dimension than the entire space $R^{n}$.

Example:

To illustrate Theorem 1 and its corollaries, we consider the simple linear system 


$$
\begin{aligned}
& \dot{\mathrm{x}}_{1}=2 \mathrm{x}_{1}+4 \mathrm{x}_{2}+\mathrm{x}_{3}-\mathrm{x}_{4}+\mathrm{u}_{2}, \\
& \dot{x}_{2}=-x_{2}+x_{4}+u_{1}+u_{2} \text {, } \\
& \dot{x}_{3}=-3 x_{3}-2 x_{4} \text {, } \\
& \dot{x}_{4}=x_{4} \text {. }
\end{aligned}
$$

The relevant matrices for this system are

$$
F=\left[\begin{array}{rrrr}
2 & 4 & 0 & -1 \\
0 & -1 & 0 & 1 \\
0 & 0 & -3 & -2 \\
0 & 0 & 0 & 1
\end{array}\right], \quad G=\left[\begin{array}{ll}
0 & 1 \\
1 & 1 \\
0 & 0 \\
0 & 0
\end{array}\right]
$$

Computing the controllability matrix $\mathscr{C}$, we have

$$
\mathscr{C}=\left[\begin{array}{rr|rr|rr|rr}
0 & 1 & 4 & 6 & 4 & 8 & 12 & 20 \\
1 & 1 & -1 & -1 & 1 & 1 & -1 & -1 \\
0 & 0 & 0 & 0 & 0 & 0 & 0 & 0 \\
0 & 0 & 0 & 0 & 0 & 0 & 0 & 0
\end{array}\right] .
$$

Since is of rank 2 , any two linearly independent vectors will suffice to form $\mathscr{S}$. For example,

$$
\mathscr{L}=\left\{\left(\begin{array}{l}
0 \\
1 \\
0 \\
0
\end{array}\right),\left(\begin{array}{l}
1 \\
1 \\
0 \\
0
\end{array}\right)\right\}
$$

The subspace of $\mathrm{R}^{4}$ generated by these vectors is the reachable set for the system. It is characterized as

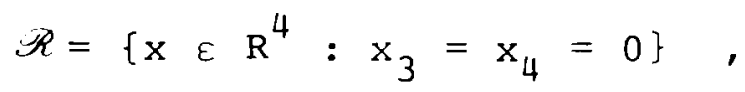

i.e., $\mathbb{R}$ is the two-dimensional set of vectors lying in the hyperplane $\mathrm{x}_{3}=\mathrm{x}_{4}=0$. 
The case of time-varying $F$ and $G$ is somewhat more complex. The basic result is

Theorem 2. Let the matrices $F(t), G(t)$ be bounded on every finite interval $t_{0} \leq t<\infty$. Then an event $\left(\tau, x^{*}\right)$ is reachable if and only if $x^{*} \varepsilon$ range $[\hat{\underline{W}}(s, \tau)]$, for some $s<\tau$, where

$$
\hat{\mathbf{W}}(\mathbf{S}, \tau)=\int_{S}^{\tau} \Phi_{F}(\tau, \sigma) G(\sigma) G^{\prime}(\sigma) \Phi_{F}^{\prime}(\tau, \sigma) d \sigma,
$$

with $\Phi(\tau, s)$ being the transition matrix of $F(t)$, i.e.

$$
\frac{\partial}{\partial t} \Phi_{F}(t, s)=F(t) \Phi_{F}(t, s), \quad \Phi_{F}(s, s)=I .
$$

Remarks:

(1) The reachable set $\mathscr{R}$ now depends upon $\tau$ and we have

$$
\mathscr{R}(\tau)=\text { range } \hat{W}\left(t_{1}, \tau\right),
$$

where $t_{1}$ is any value of $t$ for which $\hat{W}(t, \tau)$ has maximal rank.

(2) If $\mathrm{G}(\cdot)$ is zero on $(-\infty, \tau)$, we cannot have reachability of $\left(\tau, x^{*}\right)$.

(3) Making the substitution $\tau \rightarrow t, s \rightarrow 2 \tau-t$, we define a new matrix $W(\tau, t)$ which gives the analogue of Theorem 2 for controllability.

Since the rank conditions implied by remark (1) may not be easy to verify in practice, we give a simpler condition for "analytic" systems, i.e., those systems for which $F(t), G(t)$ are (real) analytic functions of $t$.

Theorem 3. Let $F(t), G(t)$ be (real) analytic functions on $t_{0} \leq t<\infty$. Define the sequence of matrix functions $Q_{i}(t)$ $\underline{\text { by }}$

$$
\begin{aligned}
Q_{0}(t) & =G(t), \\
Q_{i+1}(t) & =F(t) Q_{i}(t)-\dot{Q}_{i}(t), \quad i=0,1, \ldots, n-1 .
\end{aligned}
$$


Then the linear system $\Sigma=(F(t), G(t),-)$ is completely reachable at time $\tau$ if and only if the rank of matrix

$$
\mathscr{C}(t)=\left[\Omega_{0}(t)\left|\Omega_{1}(t)\right| \ldots \mid Q_{n-1}(t)\right]=n,
$$

for some time $t \leq \tau$.

In analogy with Theorem 1 , the reachable set for analytic systems is obtained from the matrix $\mathscr{C}(t)$ by finding the subspace spanned by a maximal set of linearly independent columns.

Example:

Consider Hill's equation with a forcing term

$$
" z(t)+(a-b(t)) z(t)=g(t) u(t),
$$

where $b(t)$ is an analytic periodic function and a is a constant. Putting $x_{1}(t)=z, x_{2}(t)=\dot{z}$, we have

$$
\begin{aligned}
& \dot{x}_{1}(t)=x_{2}(t), \\
& \dot{x}_{2}(t)=-[a-b(t)] x_{1}(t)-g(t) u(t) .
\end{aligned}
$$

It may be that the periodicity of $b(t)$ influences in some interesting way the reachability properties of (5). However, computing $\mathscr{C}(t)$ we find

$$
\mathscr{B}(t)=\left[\begin{array}{cc}
0 & -g(t) \\
g(t) & \dot{g}(t)
\end{array}\right],
$$

which implies (5) is reachable at any $t$ such that $g(t) \neq 0$. Thus, the periodic system (5) apparently has no reachability properties which are a consequence of its periodicity.

Before considering questions of observability/constructibility, it is useful and instructive to examine a number of frequently occurring variants of the basic problem considered 
above. These variants all involve imposing some restrictions either upon the desired region of state space or upon the region of admissible controls.

We begin by weakening the notion of controllability. In many cases, rather than transferring an initial state $x_{0}$ to the origin we are concerned with transferring it to some subspace $\mathscr{K} \subset \mathrm{R}^{\mathrm{n}}$. For example, $\mathscr{K}$ may represent some set of equally desirable states, a set of terminal states, etc. More precisely, we have

Definition. A system is called reachahle relative to the subspace $\mathscr{K}=\{\mathrm{x}: \mathrm{Kx}=0\}$ if for every state $\mathrm{x}_{0} \varepsilon \mathrm{R}^{+1}$, there exists a number $\bar{t}<\infty, 0 \leq t \leq \bar{t}$, such that $\operatorname{Kx}(\bar{t})=0$.

The basic result, essentially a corollary of Theorem 1, is Theorem $1^{\prime}$. The system $\Sigma=(F, G,-)$ is reachable relative to the subspace $K$ if and only if $\operatorname{rank}\left[\mathrm{KG}|\mathrm{KFG}| \cdots \mid \mathrm{KF}^{\mathrm{n}-1} \mathrm{G}\right]=\operatorname{rank} \mathrm{K}$, i.e., rank $\mathrm{K} \mathscr{C}=\operatorname{rank} \mathrm{K}$.

We have already seen that the set of controllable states forms a subspace which is generated by the columns of $\mathscr{B}$. However, in some cases we are given a particular subspace. $\mathscr{C} \subset \mathrm{R}^{\mathrm{n}}$ and would like to determine whether or not every state in $M$ is controllable. This situation is of particular importance in those processes for which we have either a priori knowledge of or influence over the initial state $\mathrm{x}_{0}$ and can assert that it belongs to a particular subspace of $\mathrm{R}^{\mathrm{n}}$.

The theorem settling the above question is

Theorem $1 "$ ". The system $\Sigma=(F, G,-)$ is conditionally controllable from a subspace $\mathscr{M}=\left\{\mathrm{x}_{0}=\mathrm{My}: \mathrm{y} \varepsilon \mathrm{R}^{\mathrm{n}}\right\}$ if and only if 
$\operatorname{rank}\left[M|G| F G|\cdots| F^{n-1} G\right]=\operatorname{rank}\left[G|F G| \cdots \mid F^{n-1} G\right]$, i.e.,

$\operatorname{rank}[M \mid \mathscr{C}]=\operatorname{rank} \mathscr{C}$.

The reader should note carefully the distinction between relative and conditional controllability. In the first instance we are concerned with whether every initial state in $R^{n}$ can be transferred to a given subspace, while the second concept involves the question of whether those states in a given subspace may be transferred to the origin.

Relative and conditional controllability have dealt with restrictions on the state space $R^{n}$ and their effect upon the controllability properties of the system. Now we consider restrictions upon the controls $u(t)$. As one might expect, imposition of constraints on the admissible inputs may have far-reaching consequences for the controllability/reachability of a system

We define the input space

$$
\begin{aligned}
\Omega^{+}= & u(t): u(t) \text { is piecewise continuous and } \\
u_{i}(t) & \geq 0, \text { for all } t\}, \quad i=1, \ldots, m .
\end{aligned}
$$

Then the problem of positive controllability is to determine whether the system $\Sigma$ is controllable/reachable with inputs from $\Omega^{+}$. It is clear that if $\Sigma$ is positively controllable, then necessarily a controllable state $x_{0}$ has the form

$$
\begin{array}{r}
\mathrm{x}_{0}=\sum_{k=1}^{\mathrm{m}} \sum_{i=0}^{\infty} \mu_{i k} F^{i}{ }^{(k),},(-1)^{i+1} \mu_{i k}>0, \\
k=1, \ldots, m, \quad i=0,1, \ldots,
\end{array}
$$

where $G^{(k)}$ is the $k^{\text {th }}$ column of $G$. Obviously, each positively controllable system is controllable but not conversely. Let us consider a few simple examples: 
i) the system

$$
\dot{\mathrm{x}}=\mathrm{u} \quad(\mathrm{x}, \mathrm{u} \text { scalars })
$$

is controllable but it is not positively controllable. The states which may be transferred to the origin by inputs from $\Omega^{+}$lie on the negative real axis.

ii) The controllable system

$$
" x=u,
$$

is also not positively controllable. The initial states $x_{0}=\{x(0), \dot{x}(0)\}$, which may be transferred to the origin by inputs from $\Omega^{+}$are given by the conditions

$$
\mathrm{x}_{0}=\mu_{0} \mathrm{~g}+\mu_{1} \mathrm{Fg}, \mu_{0}<0, \mu_{1}>0 \text {, }
$$

where $\mathrm{g}=\left(\begin{array}{l}0 \\ 1\end{array}\right), \mathrm{Fg}=\left(\begin{array}{l}1 \\ 0\end{array}\right)$. Thus, only interior points of the fourth quadrant are positively controllable to the origin.

iii) The system

$$
\begin{aligned}
& \dot{x}_{1}=x_{1} \cos \theta+x_{2} \sin \theta, \\
& \dot{x}_{2}=x_{1} \sin \theta+x_{2} \cos \theta+u, \quad 0<\theta \neq k \pi \\
& k=1,2, \ldots
\end{aligned}
$$

is positively controllable.

Various conditions have been developed to check for positive controllability. For single-input systems the most interesting is given by the following result:

Theorem 1'''. Let $[a(t)]$ _ denote the negative part of the function a(t), i.e., 


$$
[a(t)]_{-}=\left\{\begin{aligned}
a(t), & a(t)<0, \\
0, & a(t) \geq 0 .
\end{aligned}\right.
$$

Then a necessary and sufficient condition for the single-input system $\Sigma=(F, g,-)$ to be positively controllable at time $t$ is that

$$
\left[\left(z, e^{F(t-s)} g\right)\right]-\not 10,0 \leq s \leq t,
$$

for all $z,|| z||=i$.

Corollary. A single-input system $\Sigma=(F, g,-)$ is positively controllable at time $t$ if and only if

(a) The matrix $\mathscr{C}=\left[\mathrm{g}|\mathrm{Fg}| \ldots \mid \mathrm{F}^{\mathrm{n+1}} \mathrm{g}\right]$ is nonsingular.

(b) $\mathrm{F}$ has no real characteristic roots.

(c) The time $t$ is sufficiently large.

The above result shows the very interesting property that no single-input system of odd order can ever be positively controllabe. Also, we see that a single-input system cannot, in general, be positively controllable in the strict sense that any initial state may be transferred to the origin in arbitrarily short time by inputs from $\Omega^{+}$.

The multiple-input case is somewhat more delicate, requiring a surprising amount of analysis to settle with any degree of finality. A reasonable general version of the principal results is

Theorem $1^{\text {(iv) }}$. Let the input space $\Omega$ satisfy the restraints

i) $\Omega$ contains a vector in the kernel of $G$,

ii) the convex hull of $\Omega$ has nonempty interior in $R^{m}$. 
Then the following conditions are necessary and sufficient for the null-controllability of $\sum$ using inputs from $\Omega$ :

1) $\underline{\operatorname{rank} \mathscr{C}=\mathrm{n}}$,

2) there exists no characteristic vector $v$ of $F^{\prime}$ satisfying $\underline{(\mathrm{v}, \mathrm{Gu})} \leq \underline{0 \text { for all u } \varepsilon \Omega}$.

\section{Remark :}

It is important to note that the above result does not imply that the system may be driven to the origin in arbitrarily short time, only in some finite time. Arbitrary interval null-controllability requires substantially more restrictive assumptions on $\Omega$ than those given above.

Now we move on to the problem on constructibility and its counterpart, observability. Roughly speaking, the problems of observability/constructibility are concerned with state determination given information about future or past outputs. We shall distinguish two kinds of state determination problems:

i) the observation problem where the current state $x(\tau)$ is to be determined from knowledge of future outputs $\{y(s), s \geq \tau\} ;$

ii) the reconstruction problem, where the current state $x(\tau)$ is to be determined from knowledge of past outputs $\{y(s), s \leq \tau\}$.

In the first case, we observe future effects of the present state and try to determine the cause. In the second, we attempt to reconstruct the present state without complete knowledge of the state transitions.

The basic mathematical definitions for observability/ constructibility have been given above. Hence, we may pass immediately to the results. As for reachability, we begin with consideration of a constant, linear system $\Sigma=(F,-, H$.) 


$$
\begin{aligned}
& \dot{\mathrm{x}}=\mathrm{Fx} \\
& \mathrm{y}=\mathrm{Hx}
\end{aligned}
$$

Here, of course, we set $G=0$ since inputs play no essential role in observability questions. The main theorem is

Theorem 4. If $\Sigma=(\mathrm{F},-, \mathrm{H})$ is a constant linear system, then $\sum$ is completely observable/constructible if and only if the matrix

$$
\mathcal{O}=\left[H^{\prime}\left|F^{\prime} H^{\prime}\right| \ldots \mid\left(F^{\prime}\right)^{n-1} H^{\prime}\right]
$$

has rank $\mathrm{n}$.

\section{Remark:}

The observable/constructible states do not form a subspace. Just the opposite is the case: the unobservable/unconstructible states form a subspace of $\mathrm{R}^{\mathrm{n}}$. Thus, if rank $O<\overline{\mathrm{n}}$, it is not true that the linearly independent columns of $O$ generate the observable/constructible region.

\section{Trivial Example:}

Let

$$
F=\left[\begin{array}{ll}
2 & 0 \\
0 & 2
\end{array}\right], \quad H=\left[\begin{array}{ll}
0 & 1 \\
0 & 1
\end{array}\right] \text {. }
$$

Then, if $x(\tau)=\left(x_{1}^{0}, x_{2}^{0}\right)$, we have

$$
y(t)=\left[\begin{array}{ll}
0 & \\
e^{2(t-\tau)} & x_{2}^{0}
\end{array}\right]
$$

Thus, the set of states $\left\{\left(x_{1}, x_{2}\right): x_{2}=0, x_{1} \neq 0\right\}$ form an unobservable subspace for $\Sigma$. 
Less Trivial Example:

Consider the linearized dynamics of a particle in a near circular orbit in an inverse square law. force field. Assuming that the distance from the center of the force field and the angle can both be measured, we have the system matrices

$$
F=\left[\begin{array}{cccr}
0 & 1 & 0 & 0 \\
3 \omega^{2} & 0 & 0 & 2 \omega \\
0 & 0 & 0 & 1 \\
0 & -2 \omega & 0 & 0
\end{array}\right], \quad H=\left[\begin{array}{llll}
1 & 0 & 0 & 0 \\
& & & \\
0 & 0 & 1 & 0
\end{array}\right] \text {, }
$$

with $y=H x$. Here $y_{1}$ is the radial measurement, while $y_{2}$ is an angular measurement, and $\omega$ is a constant measuring the angular velocity of the particle on its circular orbit.

The observability matrix is

$$
O=\left[\begin{array}{cccccccc}
1 & 0 & 0 & 0 & 3 \omega^{2} & 0 & 0 & -6 \omega^{3} \\
0 & 0 & 1 & 0 & 0 & -2 \omega & -\omega^{2} & 0 \\
0 & 1 & 0 & 0 & 0 & 0 & 0 & 0 \\
0 & 0 & 0 & 1 & 2 \omega & 0 & 0 & -4 \omega^{2}
\end{array}\right] .
$$

This matrix has rank 4 so $\sum$ is observable/constructible.

In an attempt to minimize measurements, we might consider deleting the angular measurement $y_{2}$. In this case, $H=\left(\begin{array}{llll}1 & 0 & 0 & 0\end{array}\right)$ and

$$
=\left[\begin{array}{rrrr}
1 & 0 & 3 \omega^{2} & 0 \\
0 & 1 & 0 & -\omega^{2} \\
0 & 0 & 0 & 0 \\
0 & 0 & 2 \omega & 0
\end{array}\right]
$$

which has rank 3. Thus, without angular measurements the system is not completely observable. However, if the radial measurement is deleted, we see the $\Sigma$ will remain completely observable. 
The case of time-varying $\Sigma$ bears strong analogy to the earlier results on controllability/reachability. Specifically, we have

Theorem 5. Let $\Sigma=(F(\cdot),-, H(\cdot))$ be a real, continuous time, linear dynamical system. Then an event $(\tau, x)$ is

a) unobservable if and only if $x \in \operatorname{ker} \hat{M}(\tau, t)$ for all $t>\tau$, where

$$
\hat{M}(\tau, t)=\int_{\tau}^{t} \Phi_{F}^{\prime}(s, \tau) H^{\prime}(s) H^{\prime}(s) \Phi_{F}(s, \tau) d s \quad ;
$$

b) unconstructible if and only if $x \in \operatorname{ker} M(s, \tau)$ for all $\underline{s<\tau}$, where

$$
M(s, \tau)=\int_{S}^{\tau} \Phi_{F}^{\prime}(\sigma, \tau) H^{\prime}(\sigma) H(\sigma) \Phi_{F}(\sigma, \tau) d \sigma \quad .
$$

Here, again, $\Phi_{F}(\cdot, \cdot)$ denotes the transition matrix of $F(\cdot)$.

\section{Duality}

The reader has undoubtedly noticed a striking similarity between the definitions of the matrix functions $W$ and $M$ and the functions $\hat{W}$ and $\hat{M}$ (Theorems 2 and 5). In other words, controllability is "naturally" related (in some way) to constructibility, while observability is the natural counterpart of reachability. The most direct way to make this precise is to convert the integrand of $W$ into the integrand of $M$. For fixed $\tau$ and arbitrary real $\alpha$, the appropriate transformations are

$$
\begin{gathered}
G(\tau+\alpha) \rightarrow H^{\prime}(\tau-\alpha), \\
\Phi(\tau, \tau+\alpha) \rightarrow \Phi^{\prime}(\tau-\alpha, \tau), \\
F(\tau+\alpha) \rightarrow F^{\prime}(\tau-\alpha),
\end{gathered}
$$

Thus, we take the mirror image of the graph of each function $G(\cdot), H(\cdot), F(\cdot)$ about the point $t=\tau$ on the time axis, and 
then transpose each matrix. For controllability and constructibility, the parameter $\alpha \geq 0$, while $\alpha \leq 0$ for reachability and observability.

For constant systems, the above transformations simpliEy to

$$
\begin{aligned}
G & \rightarrow H^{\prime} \\
e^{-t F} & \rightarrow e^{-t F^{\prime}}, \\
F & \rightarrow F^{\prime} .
\end{aligned}
$$

The duality relations are clearly one-to-one, the inverses being

$$
\begin{aligned}
& H \rightarrow \mathrm{G}^{\prime}, \\
& \mathrm{F} \rightarrow \mathrm{F}^{\prime},
\end{aligned}
$$

for constant systems and

$$
\begin{aligned}
& \mathrm{H}(\tau-\alpha) \rightarrow \mathrm{G}^{\prime}(\tau+\alpha), \\
& \mathrm{F}(\tau-\alpha) \rightarrow \mathrm{F}^{\prime}(\tau+\alpha),
\end{aligned}
$$

for time-varying systems.

In view of these remarks, we can give criteria for observability and constructibility in terms of reachability and controllability and vice versa. For example, we have

Duality Theorem. The pair of matrix functions $F(t), H(t)$ define a completely observable system $\sum$ at time $\tau$ if and only if the matrix functions $F^{*}(t)=F^{\prime}(2 \tau-t), G^{*}(t)=H^{\prime}(2 \tau-t)$ define a completely reachable system $\Sigma^{*}$ at time $\tau$.

\section{B. Linearized Systems}

Armed with the above results concerning the reachability/ constructibility of linear dynamical systems, it is possible 
to begin to tackle various nonlinear problems. The most direct approach is to linearize the nonlinear system about a nominal control-state pair and to then apply the above linear theory for a local analysis. The problem here, of course, is that the results obtained pertain only to a local region in the neighborhood of the nominal trajectory and control.

Briefly, the procedure is the following: we begin with the nonlinear system

$$
\begin{aligned}
\dot{x} & =f(x, u, t), \\
y(t) & =h(x, t),
\end{aligned}
$$

Let $u *(t)$ be an admissible input and let $x^{*}(t)$ be the associated trajectory generated by Eq.(6). The dynamics and observations $(6)-(7)$ are then linearized about $\left(x^{*}, u^{*}\right)$. This yields the linearized system

$$
\begin{aligned}
\dot{x} & =F_{\left(x^{*}, u^{*}\right)}(t) x+G_{\left(x^{*}, u^{*}\right)}(t) u \\
y(t) & =H_{\left(x^{*}, u^{*}\right)}(t) x,
\end{aligned}
$$

where

$$
\begin{aligned}
& F_{\left(x^{*}, u^{*}\right)}(t)=\left.\frac{\partial f}{\partial x}(x, u, t)\right|_{x=x^{*}(t)}, \\
& u=u^{*}(t) \\
& G_{\left(x^{*}, u^{*}\right)}(t)=\left.\frac{\partial f}{\partial u}(x, u, t)\right|_{x}=x^{*}(t) \\
& H_{\left(x^{*}, u^{*}\right)}(t)=\left.\frac{\partial h}{\partial x}\right|_{x=x^{*}(t)},
\end{aligned}
$$


Clearly, the results obtained from such an analysis make sense only if $i)$ the functions $f$ and $h$ are sufficiently smooth to justify the linearization and $i i)$ we confine our attention to sufficiently small neighborhoods of the nominal trajectory and control. Here "sufficiently small" must be interpreted in terms of the analytic properties of $f$ and $h$, i.e., how close they are to being linear and their degree of smoothness. To illustrate the above ideas, we begin with

\section{Definition 5. Consider the process}

$$
\begin{aligned}
& \dot{x}=f(x, u, t)=F x+G u+\ldots \\
& y=h(x, t)=H x+\ldots
\end{aligned}
$$

near $x^{*}=0, u^{*}=0$ (here we use the hypotheses $f(0,0, t)=$ $h(0, t)=0)$.

The process is locally controllable if for each $\bar{x}$ in some neighborhood of the origin, there exists a piecewise continuous control $u(t), 0 \leq t \leq \tau$, such that the system may be transferred to the state $\bar{x}$ from the origin in time $\tau, \tau$ sufficiently small.

The process is locally observable if for each sufficiently small piecewise-continuous control $u(t)$ on $0 \leq t \leq \tau$, the equality

$$
h\left(x_{1}(t), t\right)=h\left(x_{2}(t), t\right)
$$

implies

$$
x_{1}(t)=x_{2}(t), \quad 0 \leq t \leq \tau, \quad \tau \text { sufficiently small }
$$

The basic result on local controllability and observability is that the global linear results are sufficient for the local nonlinear results, i.e.,

Theorem 6. The process (*) is locally controllable is $\operatorname{rank}\left[G|F G| \ldots \mid F^{n-1} G\right]=n ;$ 
it is locally observable if

$$
\operatorname{rank}\left[H^{\prime}\left|F^{\prime} H^{\prime}\right| \ldots \mid\left(F^{\prime}\right)^{n-1} H^{\prime}\right]=n
$$

Example [7]:

Consider a very crude model of a national economy in which we look at only the gross national product $Y$, the income arising from the consumption of goods and services $C$, and the investment income $I$. We also include governmental expenditures $G$ to complete the model.

The first balance equation is

$$
Y=C+I+G
$$

We next note that $C$ depends upon disposable income $\mathrm{Y}_{\mathrm{D}}=\mathrm{a}\left(\mathrm{Y}-\mathrm{T}_{\mathrm{X}}\right)+\mathrm{b}$, where $\mathrm{T}_{\mathrm{X}}=$ tax rate with $\mathrm{a}$ and $\mathrm{b}$ constants, $0<\mathrm{a}<1, \mathrm{~b}<0$. Also, I depends upon the basic interest rate $r, I=I(r)$. Thus, the three equations

$$
\begin{aligned}
& Y=C+I+G, \\
& C=a\left(Y-T_{x}\right)+b, \\
& I=I(Y),
\end{aligned}
$$

describe the controlled macroeconomy.

Now consider the economic model near some state $\mathrm{Y}_{0}, \mathrm{C}_{0}$, $\mathrm{I}_{0}$, with the corresponding controls $\mathrm{G}_{0}, \mathrm{~T}_{\mathrm{x}_{0}}, \mathrm{r}_{0}$. Assume the interest rate is changed so that the investment is changed to

$$
I=I_{0}+i
$$

similarly

$$
\mathrm{Y}=\mathrm{Y}_{0}+\mathrm{Y}, \mathrm{C}=\mathrm{C}_{0}+\mathrm{C} \text {. }
$$


Assuming a static equilibrium at each stage of the time-varying process (what might be called an adiabatically-varying economy), we obtain the relations between the small variations $y, c$, and i :

$$
\begin{aligned}
& y=c+i, \\
& c=a y,
\end{aligned}
$$

These are static equilibrium conditions at each instant.

Suppose the economy were not in equilibrium at some instant t. At such a time

$$
c+i-y \neq 0 \text { or ay-c } \neq 0 \text {, }
$$

and we postulate that the economy tends towards the corresponding equilibrium state according to the dynamical equations

$$
\begin{aligned}
& \frac{d c}{d t}=\beta(a y-c)+\ldots, \\
& \frac{d y}{d t}=\alpha(c+1-y)+\ldots
\end{aligned}
$$

Here, $\alpha>0, \beta>0$ are constants describing the dynamics of the economy which may be nonlinear as indicated by the terms $+\ldots$. The observed output is $y$ and so the appropriate matrices for this linearized problem are

$$
F=\left[\begin{array}{cc}
-\beta & \beta a \\
\alpha & \alpha
\end{array}\right], \quad G=\left(\begin{array}{l}
0 \\
\alpha
\end{array}\right), \quad H=\left(\begin{array}{ll}
0 & 1
\end{array}\right)
$$

According to Theorem 6 , the local controllability condition is

$$
\operatorname{rank}\left(\begin{array}{cc}
0 & \alpha \beta a \\
\alpha & -\alpha^{2}
\end{array}\right)=2,
$$


while the observability condition is

$$
\operatorname{rank}\left(\begin{array}{cc}
0 & \alpha \\
1 & -\alpha
\end{array}\right)=2 \text {. }
$$

Thus, this system will be locally controllable and observable if $\alpha \beta a \neq 0$.

\section{Bij.inear (Multilinear) systems}

The simplest class of nontrivial nonlinear systems, and the only one for which substantial analytic advances have been made, are systems which are linear in the state and control separately, but not jointly, i.e., bilinear systems. A simple scalar example of a system of this sort is

$$
\dot{x}=a x+b u+c x u \text {. }
$$

Obvious extensions to the case when the system is multilinear in the state and/or control will be pursued briefly later.

In view of the relationship between the solution of a bilinear system such as (8), and a time-varying linear system of the form $\dot{x}=A(t) x+B(t) u$, together with the well known connections of the latter type of system with the Lie-algebraic methods of Wei and Norman [20], one would conjecture that the controllability/observability properties of bilinear processes will be essentially algebraic in nature. Thus, the algebraic flavor so evident for linear problems seems also to be an intrinsic feature of the few totally nonlinear problems that have been studied and, as a result, it seems likely that substantial progress in analyzing controllability/observability properties of nonliner systems will rely upon a thorough study of the algebraic structures involved.

For ease of exposition, but without loss of generality, for the most part we shall consider homogeneous-in-the-state bilinear systems of the type 


$$
\dot{\mathrm{x}}=\mathrm{Fx}+\mathrm{Nxu}, \quad \mathrm{x}(0)=\mathrm{x}_{0},
$$

where $\mathrm{x}$ and $\mathrm{u}$ are $\mathrm{n}, \mathrm{m}$-dimensional vectors, respectively and we use the shorthand notation

$$
\mathrm{Nxu} \triangleq \sum_{i=1}^{\mathrm{m}} \mathrm{N}_{i} \mathrm{xu}_{i}(t),
$$

where $u_{i}(t)$ is the $i^{\text {th }}$ component of the vector $u$. The matrices $F, N_{i}$ are assumed constant. It is easily seen that the solution of (9) is given in the form

$$
x(t)=\Gamma(t) x_{0},
$$

where $\Gamma(t) \varepsilon \mathscr{G}_{n}$, the set of real, nonsingular $n \times n$ matrices, $t \geq 0$. To see that the vector system (9) also includes systems of the form

$$
\dot{z}=A z+B v+C z V,
$$

let $\mathrm{F}$ and $\mathrm{N}_{\mathrm{i}}$ be defined by adding a single extra row and column to $A$ and $C_{i}$, respectively

$$
F=\left[\begin{array}{ll}
0 & 0 \\
0 & A
\end{array}\right], \quad N_{i}=\left[\begin{array}{cc}
0 & 0 \\
B & \\
(i) & C_{i}
\end{array}\right],
$$

where $B^{(i)}$ is the $i^{\text {th }}$ column of $B$. Now define

$$
x=\left[\begin{array}{l}
1 \\
z
\end{array}\right], \quad u=v \text {. }
$$

From (10), the first intrinsic property of homogeneousin-the-state bilinear systems appears: the origin is never controllable! Thus, a more convenient state space for this type of problem is the "punctured" space $\mathrm{R}^{\mathrm{n}}-\{0\}$. 
Another consequence of $(10)$ is that reachability and controllability properties of (9) are directly connected to the analogous properties of the matrix system

$$
\dot{\mathrm{X}}=\mathrm{FX}+\mathrm{NXu},
$$

where the state space is taken to be $\mathscr{G}_{\mathrm{n}}$ and

$$
N X u \triangleq \sum_{i=1}^{m} N_{i} X u_{i}(t)
$$

It suffices to study system (11) under the condition that $\mathrm{X}(0)=\mathrm{I}$, since if we consider any other $\mathrm{x}_{0} \varepsilon \mathscr{G}_{\mathrm{n}}$, then the reachable set at time $T$ equals

$$
\left\{\mathrm{X} \varepsilon \mathscr{G}_{\mathrm{n}}: \mathrm{X}=\Gamma \mathrm{X}_{0} \text {, with } \Gamma \varepsilon \text { reachable set of (11) at } \mathrm{T}\right\} \text {. }
$$

If we let $\mathscr{R}(I)$ denote the set of points reachable from the identity at any time $t \geq 0$, then it can be shown that if $\mathscr{R}(I)$ is a transitive group for (11) on $\mathrm{R}^{\mathrm{n}}-\{0\}$, i.e., if $\mathscr{R}(I)$ is such that for all $\mathrm{x}, \overline{\mathrm{x}} \varepsilon \mathrm{R}^{\mathrm{n}}-\{0\}$, there exists a $\Gamma \in \mathscr{R}(I)$ such that $\mathrm{x}=\Gamma \overline{\mathrm{x}}$, then (9) is completely controllable on $\mathrm{R}^{\mathrm{n}}$ - $\{0\}$. This result establishes the connection between studying (11) in order to obtain results on the controllability/ reachability of (9).

Some additional definitions will be needed to concisely state the results to follow.

Definition 6. Given two $n \times n$ matrices $A, B$, their Lie product is defined as

$$
[\mathrm{A}, \mathrm{B}] \stackrel{\triangle}{=} \mathrm{AB}-\mathrm{BA}
$$

Definition 7. A Lie algebra $\&$ in the space of $n \times n$ matrices is a linear subspace of $n \times n$ matrices which is closed under the Lie product operation. 
Definition 8 . Given a subset $\mathscr{S}$ of the set of $\mathrm{n} \times \mathrm{n}$ matrices, the Lie algebra generated by $\mathscr{S}$ is the smallest Lie algebra containing $\mathscr{f}$.

Definition 9. Given a Lie algebra $£$ in the set of $n \times n$ matrices, we define

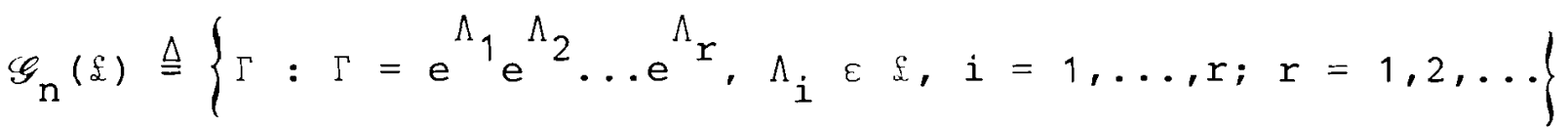

$\mathscr{G}_{\mathrm{n}}(\dot{i})$ is called the connected Lie group associated with $\dot{\Sigma}$

\section{Notation:}

We let $\aleph_{1}=$ the Lie algebra generated by $\left\{\mathrm{F}, \mathrm{N}_{1}, \ldots, \mathrm{N}_{\mathrm{m}}\right\}$.

The principal result concerning controllability (or reachability) of the homogeneous system (11) is

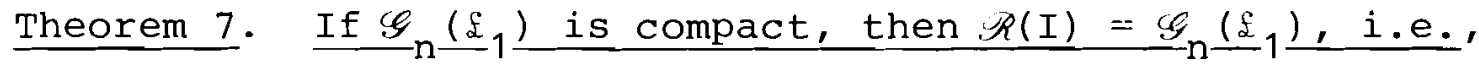

$$
\mathscr{R}(I)=\left\{\Gamma: \Gamma=e^{\Lambda_{1}} \mathrm{e}^{\Lambda_{2}} \ldots \mathrm{e}^{\Lambda_{\mathrm{r}}}, \Lambda_{\mathrm{i}} \in £_{1}, i=1, \ldots, \mathrm{r} ; \mathrm{r}=1,2, \ldots\right\} \text {. }
$$

A much simpler form of this result is valid for vector systems of the form

$$
\dot{x}=\left(F+\sum_{i=1}^{m} N_{i} v_{i}(t)\right) x(t)+\sum_{i=1}^{r} u_{i}(t) G^{(i)} .
$$

Here the control has been separated into two parts: one part (the vector $v(t)$ ) is associated with the purely bilinear part of the system, while the other part (the vector $u(t)$ ) is associated with the purely linear part. The elements $G^{(i)}$ are the columns of the linear input matrix $G, i=1, \ldots, r$. The principal result is

Theorem 8. The reachable set for the system (12) starting at $x=0$ at time $t=0$ is the vector space generated by the set $\mathscr{S}=\left\{\mathrm{L}_{\mathrm{i}}^{\mathrm{k}}{ }^{(\mathrm{i})}\right\}$, where the $\mathrm{L}_{i}$ are a basis for the Lie algebra $f, k=0,1, \ldots$.

Theorem 8 is the natural generalization of Theorem 1 as is easily seen by setting all $\mathrm{N}_{i}=0, i=1, \ldots, \mathrm{m}$. In this event the Lie algebra generated by $\left\{F, N_{i}, \ldots N_{m}\right\}$ is just $F$ 
itself, hence a basis is also F. Thus, the reachable set is the vector space generated by the set $\mathscr{S}=\left\{F^{k_{G}}{ }^{(i)}\right\}, k=0,1, \ldots$, $i=1, \ldots, r$. But, by the Hamilton-Cayley theorem it suffices to restrict $\mathrm{k}$ to the range $\mathrm{k}=0,1, \ldots, \mathrm{n}-1$. Hence, the vectors of the set $\mathscr{f}$ coincide with those of the controllability matrix 6 of Theorem 1.

We turn now to questions of observability and constructibility for the homogeneous system (11). Assume that the system output is given by

$$
y(t)=h(X(t)),
$$

where $x(t) \in \mathscr{G}_{\mathrm{n}}$ for all $t$. The exact nature of the output map $\mathrm{h}(\cdot)$ is not essential. We assume nothing about the output space - it is just a set. The critical assumption is that there exist subgroups $\mathrm{H}_{\ell}$ and $\mathrm{H}_{r}$ of $\mathscr{G}_{\mathrm{n}}$ such that $\mathrm{h}\left(\mathrm{x}_{1}\right)=\mathrm{h}\left(\mathrm{x}_{2}\right)$ if and only if $\mathrm{H}_{1} \mathrm{X}_{1} \mathrm{H}_{2}=\mathrm{x}_{2}$, where $\mathrm{H}_{1}{ }^{\prime} \varepsilon \mathrm{H}_{\ell}, \mathrm{H}_{2} \varepsilon \mathrm{H}_{\mathrm{r}}$. Under this assumption, $h(X)$ identifies $x$ only to within conjugation by elements of $\mathrm{H}_{\ell}$ and $\mathrm{H}_{r}$. We call such systems homogeneous. In such a set-up, the observation of $y(t)$, even over an extended period of time, can at most determine $x$ only up to a right multiplication by an element of $\mathrm{H}_{r}$. Thus, we may as well regard the system as evolving on the coset space $\mathscr{G}_{\mathrm{n}} / \mathrm{H}_{\mathrm{r}}$. The question we pose is whether or not the observation of $y(t)$ and knowledge of $u(t)$ over the interval $[0, \infty]$, serves to identify uniquely an element of $\mathscr{G}_{\mathrm{n}} / \mathrm{H}_{\mathrm{r}}$ as an initial state. The theorem which answers this query is

Theorem 9. Let $\mathscr{R}(I)$ denote, as before, the set of states of (11) reachable from $I$ and suppose that $\mathscr{R}(I)$ is a group.

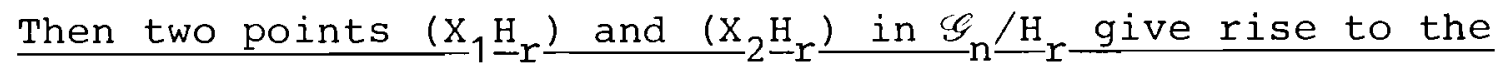
same input/output map if and only if for each $\mathrm{Z} \varepsilon \mathscr{R}(I)$, there exists an $\mathrm{H}_{1}(\mathrm{Z}) \quad \varepsilon \mathrm{H}_{\ell}$ such that

$$
\mathrm{z}^{-1} \mathrm{H}_{1}(\mathrm{z}) \mathrm{z}\left(\mathrm{X}_{1} \mathrm{H}_{\mathrm{r}}\right)=\left(\mathrm{X}_{2} \mathrm{H}_{\mathrm{r}}\right)
$$


If we let

$$
\mathscr{P}=\left\{\mathrm{X}: \mathrm{Z}^{-1} \mathrm{XZ} \in \mathrm{H}_{\ell^{\prime}} \text { for all } \mathrm{Z} \in \mathscr{R}(I)\right\},
$$

then any two elements of the form $\left(\mathrm{X}_{1} \mathrm{H}_{\mathrm{r}}\right)$ and $\mathrm{P}_{1}\left(\mathrm{X}_{1} \mathrm{H}_{\mathrm{r}}\right)$ with $\underline{P}_{1} \in \mathcal{E}$ are not distinguishable.

By specializing the output map $h(\cdot)$ and imposing various topologies on the output set, more specific criteria for constructibility may be obtained. However, it is clear from Theorem 9 that the essential issue is a purely algebraic one, having no dependence on analytical considerations. As an illustration, let us assume that the system under study is

$$
\dot{\mathrm{x}}=\mathrm{Fx}+\mathrm{Nxu}+\mathrm{Gu}
$$

and that the outputs $\mathrm{y}=\mathrm{Hx}(\mathrm{t})$ are linear and belong to the vector space $R^{p}$. Then the following theorem may be given:

Theorem 10. Define the matrix sequence

$$
Q_{1}=H \quad, \quad Q_{i}=\left(\begin{array}{l}
Q_{i-1} F \\
---- \\
Q_{i-1} N
\end{array}\right), \quad i=1,2, \ldots .
$$

Then the subset of unobservable (or unconstructible) gtates of Eq. (13)

(a) forms the largest subspace invariant under $\mathrm{F}$ and $\mathrm{N}$ and contained in $\mathscr{H}(\mathrm{G})$;

(b) may be expressed as

$$
\mathscr{N}\left[\begin{array}{c}
Q_{1} \\
-1 \\
\Omega_{2} \\
\hdashline \vdots \\
\hdashline- \\
Q_{n}
\end{array}\right]
$$

where $\mathscr{N}(\mathrm{A})$ denotes the null space of the matrix $\mathrm{A}$. 
The foregoing results regarding bilinear systems may be interpreted as statements concerning the class of "physically interesting" multilinear systems by virtue of the following fundamental result:

Theorem 11[10]. The conditions under which the canonical state set of a given multilinear input/output function can be constructed in a finite number of steps (i.e., is finitedimensional are identical to those under which the same function may be realized by a dynamical system with a bilinear internal structure.

As a consequence of this remarkable result, there is no added generality in assuming that a given experimental set-up is described by a multilinear, rather than bilinear system. If the given experimental data can be explained by any finitedimensional multilinear state space model, then it can be explained by a bilinear model. Of course, this does not mean that a totally nonlinear model is equivalent to a bilinear one but it does substantially restrict the cases which need be considered. As an example, by introduction of a sufficient number of new variables, and polynomial model may be replaced by a multilinear structure which, by Theorem 11, must then be mathematically equivalent (modulo the finiteness restriction) to a bilinear system.

D. Nonlinear Systems

As one might conjecture from the results on multilinear systems, the reachability problem for general nonlinear systems of the form

$$
\dot{x}=f(x, u),
$$

may be studied by examining the Lie algebra generated by the vector field $f$. Unfortunately, in the general case this problem cannot be reduced to matrix computations as was done above; however, in principle the same techniques apply and once some structure is imposed upon $\mathrm{f}$ various computational approaches 
may be employed. A detailed discussion of these matters requires a degree of mathematical sophistication beyond the bounds of this elementary survey, so we shall refer the reader to [11] for further information. It is of some interest to note, however, that the observability problem for nonlinear systems has received very little attention in the literature with the exception of the brief discussion in [3]

On the above note, we conclude this all too brief survey of methodology and return to the question of its relevance to applied systems analysis - IIASA-style.

\section{System-Theoretic Problems at IIASA}

This section examines several problems that have been described in earlier IIASA publications from the viewpoint of the methodology discussed above. It is not intended that any of these examples be extensively pursued in the future nor is it of particular interest that these problems represent the "state-of-the-art" in the areas they model. What is important, though, is that they have been seriously proposed as such models. Our aim is only to point out that there have been in the past (and presumably there will continue to be) problems of IIASA interest which possess definite system-theoretic overtones, regardless of whether or not these aspects have been recognized. Ideally, this brief survey of "hometown" problems will be sufficient motivation for future work.

Many of the problems that follow were originally presented within the context of an optimization process. In accordance with the general principle that system structure is more or less independent of externally imposed criteria, we abstract here only those features which do not relate to the particular objective function initially chosen.

\section{A. Renewable Resources Management [12]}

A major ecological problem is to develop strategies.for the development of resources. The real issue here, as pointed out in [12], is to devise sensible (and implementable) har- 
vesting policies in the face of uncertainties regarding the dynamics of the process and within the context of an everchanging political and economic environment. This question properly belongs within the domain of adaptive control theory; however, various versions of it may be considered as reachability/constructibility questions for which the above methodology may yield some insight.

Suppose we have a resource system whose state at time $t$ may be described by a vector $x(t)$. A simplified version of the dynamics of this system as adapted from [12] is

$$
\dot{x}(t)=S x(t)+B x(t-\tau)-C x(t) E(t),
$$

where $\mathrm{S}$. is a matrix of survival rates, $\mathrm{C}$ is a matrix of "catchability coefficients", B is a matrix describing the growth of current population which is dependent upon the state of the system $T$ units in the past, and $E(t)$ is a vector of total harvesting effort (control) or some other measure of exploitation intensity.

With the exception of the time-lag term involving $x(t-\tau)$, the above problem is seen to be a bilinear control process of the type studied in Part II. If we assume that the time-lag $\tau$ is small compared with the value of $t$ (i.e., the system has been in operation for a large time interval), then we may approximate $\mathrm{x}(\mathrm{t}-\tau)$ as

$$
x(t-\tau) \cong x(t)-\tau \dot{x}(t)+\frac{\tau^{2}}{2} " x(t) \ldots .
$$

Keeping only terms of order $\tau^{2}$, the above system may be approximated by a modified bilinear process.

Some of the questions which one could attack with the techniques given in Part II include:

i) description of the reachable state space at time $t$, given constraints on the admissible catch effort $E(t)$, i.e., $E(t) \varepsilon \mathscr{S}(t)$;

ii) sensitivity of the reachable set to changes in system parameters; 
iii) if it is assumed that $E(t)$ is related in some way to taxation and investment rates, a description of the reachable set in terms of these "auxiliary" controls.

Problems of observability/constructibility also enter into the above framework. In fact, according to [12]: "in most cases $x(t)$ is not directly observable...usually it is only possible to measure the total catch $C E(t) x(t)$ and the effort $E(t) . "$ Thus, we see that the observability techniques may provide vital information to the system manager in enabling him to accurately measure the state of the system before deciding upon his action $E(t)$. Alternatively, system-theoretic techniques may show the manager that there are inherent structural features of the system that will forever prevent him from obtaining complete information about the process, even in the absence of stochastic effects. Such knowledge may suggest alternate models and/or a restructuring of the basic measurement process.

In many instances, some or all of the components of the system matrices S, B, and C may be subject to stochastic perturbations having known or unknown distribution functions. In the first case, the preceding methodology may be used in an expected value sense; in the latter case, one is faced with an adaptive process whose methodological treatment transcends the limitations and modest aims of this report. The key point, however, is that the introduction of randomness into the process may complicate the computational aspects of the situation but it presents no new conceptual hurdles. Thus, a thorough understanding of the deterministic methodology will, with sufficient computing power, enable one to deal also with the stochastic case. This is a point that has been repeatedly emphasized in earlier IIASA deliberations.

A somewhat more complicated version of the above problem is given in [13] for the determination of harvesting strategies for salmon. In this problem, the linear time-lag term $\mathrm{Bx}(t-\tau)$ is replaced by a nonlinear curve, the so-called 
Ricker model, without a time-lag and $S$ is taken to be zero, i.e., we have the dynamics (after some algebraic re-arrangement)

$$
S_{t+1}=e^{-\alpha\left(1-S_{t}\right)} s_{t} z_{t}
$$

where $s_{t}$ is the salmon stock level at time $t, \alpha$ is a parameter reflecting the net stock productivity, and $z_{t}=1 /\left(1-u_{t}\right)$, with $u_{t}$ representing the net exploitation rate.

By expanding the exponential term as

$$
e^{-\left(1-s_{t}\right)} \cong 1-\left(1-s_{t}\right)+\frac{\alpha^{2}}{2}\left(1-s_{t}\right)^{2}+\ldots
$$

and truncating at some appropriate point, the salmon model becomes a polynomial system with control entering linearly. The reachability structure of such systems may be studied by several means: linearization, conversion to a multilinear problem by introduction of additional state variables, or directly by Lie-algebraic methods.

\section{B. Water Reservoir Regulation [14]}

A problem that arises in most water basin networks throughout the world is that of regulating the flow through various dams in the network in order that the entire system behave in some prescribed fashion. Generally, this problem is complicated by the presence of stochastic inflows to the network due to rainfall and underground water run-off. In addition, the conflicting objectives of the various water users must be taken into account. Here we shall consider only the reachability/constructibility questions involving the physical water basin network itself.

A simple example of a problem of this genre is depicted in Figure 4. 


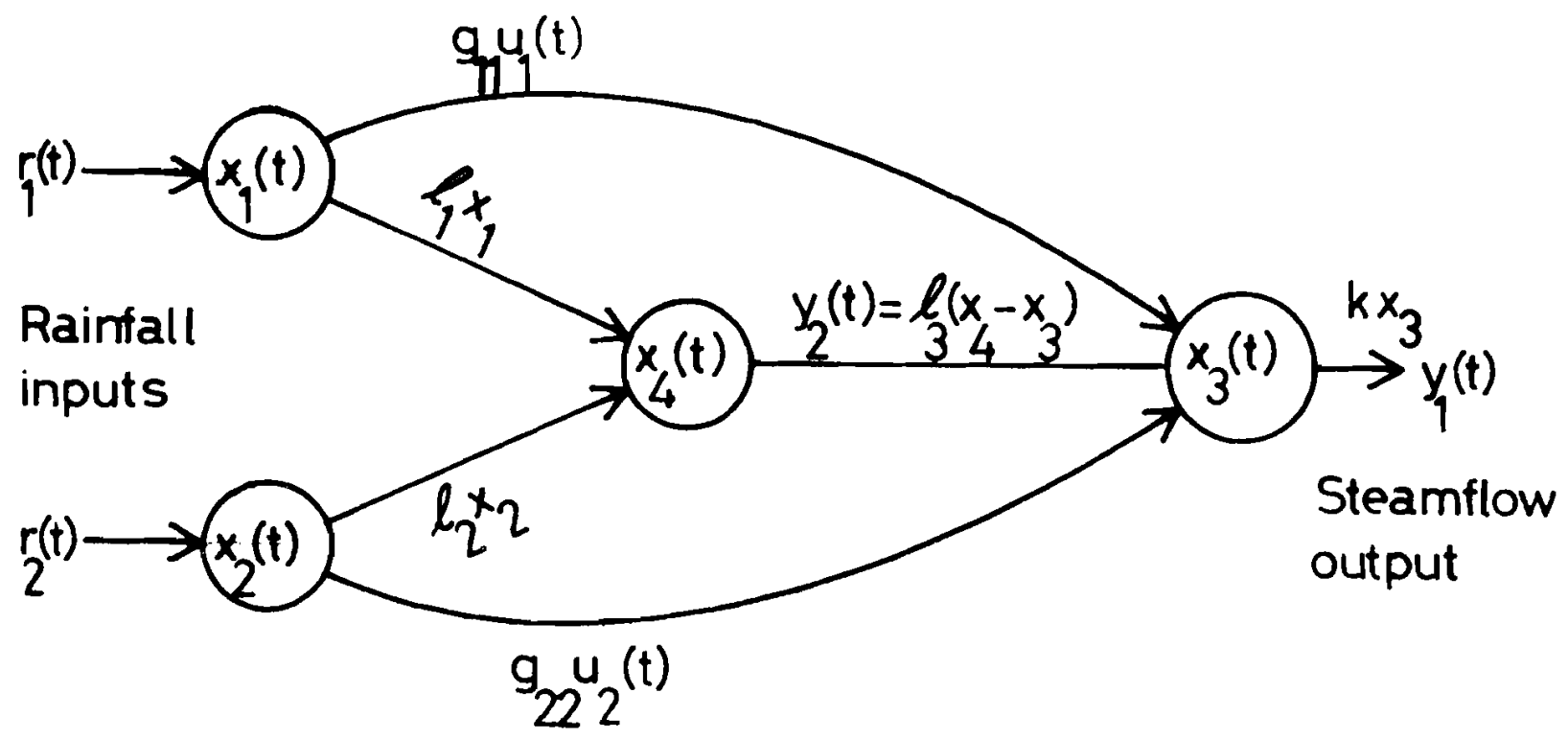

Figure 4. Water Reservoir Network

In Figure $4, r_{1}(t)$ and $r_{2}(t)$ are the rainfall inputs, the states of surface storage at locations $1-3$ are $x_{1}(t), x_{2}(t)$, $x_{3}(t)$, respectively, while the state of groundwater storage (including infiltration) is $x_{4}(t)$. The constants $l_{1}$ and $l_{2}$ are for infiltration. The expression $\ell_{3}\left(x_{4}-x_{3}\right)$ signifies the exchange between the river and groundwater. The system outputs $y_{1}, Y_{2}$ are the streamflow output and the contribution of groundwater, respectively.

The continuity equations for this problem are

$$
\begin{aligned}
& \dot{x}_{1}=-l_{1} x_{1}+r_{1}-g_{11} u_{1}, \\
& \dot{x}_{2}=-l_{2} x_{2}+r_{2}-g_{22} u_{2}, \\
& \dot{x}_{3}=l_{3}\left(x_{4}-x_{3}\right)-k x_{3}+g_{11} u_{1}+g_{22} u_{2}, \\
& \dot{x}_{4}=l_{1} x_{1}+l_{2} x_{2}-l_{3}\left(x_{4}-x_{3}\right) .
\end{aligned}
$$


The outputs are

$$
\begin{aligned}
& y_{1}=k x_{3} \\
& y_{2}=l_{3}\left(x_{4}-x_{3}\right)
\end{aligned}
$$

In vector-matrix form, we have

$$
\begin{aligned}
& \dot{x}(t)=\mathrm{Fx}+\mathrm{Gu}+\mathrm{r}(\mathrm{t}), \quad \mathrm{x}(0)=\mathrm{c}, \\
& \mathrm{y}(\mathrm{t})=\mathrm{Hx},
\end{aligned}
$$

where

$$
\begin{aligned}
& F=\left[\begin{array}{cccc}
-\ell_{1} & 0 & 0 & 0 \\
0 & -\ell_{2} & 0 & 0 \\
0 & 0 & -\ell_{3} & \ell_{3} \\
\ell_{1} & \ell_{2} & \ell_{3} & -\ell_{3}
\end{array}\right] \\
& G=\left[\begin{array}{cc}
-g_{11} & 0 \\
0 & -g_{22} \\
g_{11} & g_{22} \\
0 & 0
\end{array}\right], \quad r(t)=\left[\begin{array}{l}
r_{1}(t) \\
r_{2}(t) \\
0 \\
0
\end{array}\right], \quad H=\left[\begin{array}{llll}
0 & 0 & k & 0 \\
0 & 0 & -l_{3} & l_{3}
\end{array}\right]
\end{aligned}
$$

By virtue of the closed form expression

$$
x(t)=e^{F t} c+\int_{0}^{t} e^{F(t-s)}[G u(s)+r(s)] d s,
$$

we see that the reachability/constructibility features of the above process are independent* of the rainfall input $r(t)$. Thus, for purposes of analysis, there is no loss of generality in assuming $r(t) \equiv 0$. (Intuitively, this is due to the tacit assumption that $u(t)$ can be made arbitrarily large. In the more realistic case when $0 \leq u_{i}(t) \leq U$, a more refined analysis is required.)

* independent in the sense that results for the case $r(t) \not z 0$ may be obtained from the $r=0$ case simply by adding the unknown vector function $-\int_{0}^{t} e^{F(t-s)} r(s) d s$ to $x$. 
It is an amusing exercise to apply the techniques of Part II to the above system to discover what is already evident from Figure 4; namely, that the system is completely reachable as long as $g_{11} \neq 0, g_{22} \neq 0$, and is also completely constructible. However, note the central role played by groundwater interchange with surface storage in the determination of constructibility, i.e., if $\ell_{3}=0$ (no interchange) then no amount of streamflow observation gives any information about groundwater storage.

Other water resource problems of a similar nature may be found in the IIASA reports [15] and [16].

C. National Settlement Planning [17]

A number of IIASA urbanologists have been concerned with the question of developing national settlement strategies subject to constraints on resources, immigration quotas, and the like. Several different approaches have been proposed for dealing with this sort of problem, some of them falling into the basic framework considered in this paper. We describe one of these "system-theoretic" approaches, first presented in [17]. The essential aspect of this model is to promote a desired migratory process by differential stimulation of the employment market on the part of the government.

The state equations for the model are

$$
\begin{aligned}
& \mathrm{x}(\mathrm{t}+1)=\mathrm{Kx}(\mathrm{t})+(I-M) \mathrm{v}(\mathrm{t}), \\
& \mathrm{v}(\mathrm{t}+1)=\mathrm{Mv}(t)+\mathrm{u}(\mathrm{t})+\mathrm{z}(\mathrm{t}),
\end{aligned}
$$

where the vector $\mathrm{x}(t) \in \mathrm{R}^{\mathrm{n}}$ represents the population distribution at time $t, v(t) \varepsilon R^{n}$ is the distribution of job vacancies at time $t, u(t) \varepsilon R^{n}$ is the distribution of government stimulated job vacancies and $z(t) \varepsilon R^{n}$ is the distribution of spontaneously occurring vacancies. The matrix $K$ is a diagonal matrix whose elements reflect the natural population growth rates within a region, while $M$ is a migration matrix with elements $m_{i j}$ being the probability that a job vacancy 
in region $j$ will be filled by someone living in region $i$, $i, j=1, \ldots, n$. The problem, of course, is to choose $u(t)$ so that $x(t)$ (and possibly $v(t)$ ) follows some desired course. The budgetary and fixed immigration constraints on the choice of $u(t)$ are given by

$$
\begin{aligned}
\text { i) } & u(t) \geq 0, \\
\text { ii) }(u(t), r(t)) \leq b, & \\
\text { iii) } & || u(t)|| \leq \hat{u}, \quad t=1,2, \ldots, T .
\end{aligned}
$$

Here (,) denotes the vector inner product, while ||$\cdot||$ is some appropriate norm (e.g., $\left.\ell_{1}\right)$, with $r(t)$ being a function giving the total financial resource available to be offered regionally by the government at period $t$, b being the total budget available.

By introducing the new vectors

$$
\begin{array}{r}
s(t)=\left[\frac{0}{u(t)}\right], \quad w(t)=\left[\frac{x(t)}{v(t)}\right], \quad y(t)=\left[\frac{0}{z(t)}\right], \\
s, w, y \in R^{2 n,},
\end{array}
$$

it is possible to rewrite the above model in the form

$$
w(t+1)=F w(t)+G s(t)+y(t),
$$

where

$$
F=\left[\begin{array}{c|c}
K & I-M \\
\hline 0 & M
\end{array}\right], \quad G=\left[\begin{array}{l|l}
0 & 0 \\
\hline 0 & I
\end{array}\right] \text {. }
$$

The above constraints restrict the region of admissible inputs $s(t)$. Actually, on the basis of more detailed analysis, for purposes of determining reachable sets it suffices to replace inequalities $i i)$ and $i i i)$ by the corresponding equality. (Physically, this fact is fairly obvious but requires a surprising amount of analysis to prove.) 
The usual questions surrounding reachability may now be studied with the above "standard" model. As with the previous water example, the forcing term $y(t)$, corresponding to the spontaneously arising jobs, plays no structural role in the reachability analysis.

The model given in [17] included no inherent notion of observability. However, it is reasonable to suppose that in many cases the vectors $x(t), v(t)$ will not be available for direct measurement and, if the migration strategy is to be based upon the current state of the system rather than upon an "ostrich-like" open-loop policy, then observability/constructibility questions will arise. A more detailed pursuit of this point will likely prove necessary before a model of the above type could be used in practice with any degree of confidence.

\section{Transportation Systems [18]}

Problems involving the regulation of automative traffic flow in urban areas seem tailormade for the type of methodology we have been discussing. Many, if not most, of the mathematical models surveyed in [18] involve systems in which the dynamics of the traffic flow are linear, with the primary question being whether or not it is possible to reach a state of "undersaturation" (normal flow), from a state of "oversaturation" (rush-hour congestion) within a specified time period utilizing various regulation policies (usually control of freeway on-off ramps by traffic lights). Obviously, this is a reachability problem. If the control policy is to be generated by feedback, then observability/constructibility considerations enter when one analyzes the possibility of determining the current state of traffic based upon measurements being received from various sensing devices. Here we present one of the examples from [18] to illustrate the main ideas.

Consider the rectangular traffic network depicted in Figure 5 . 


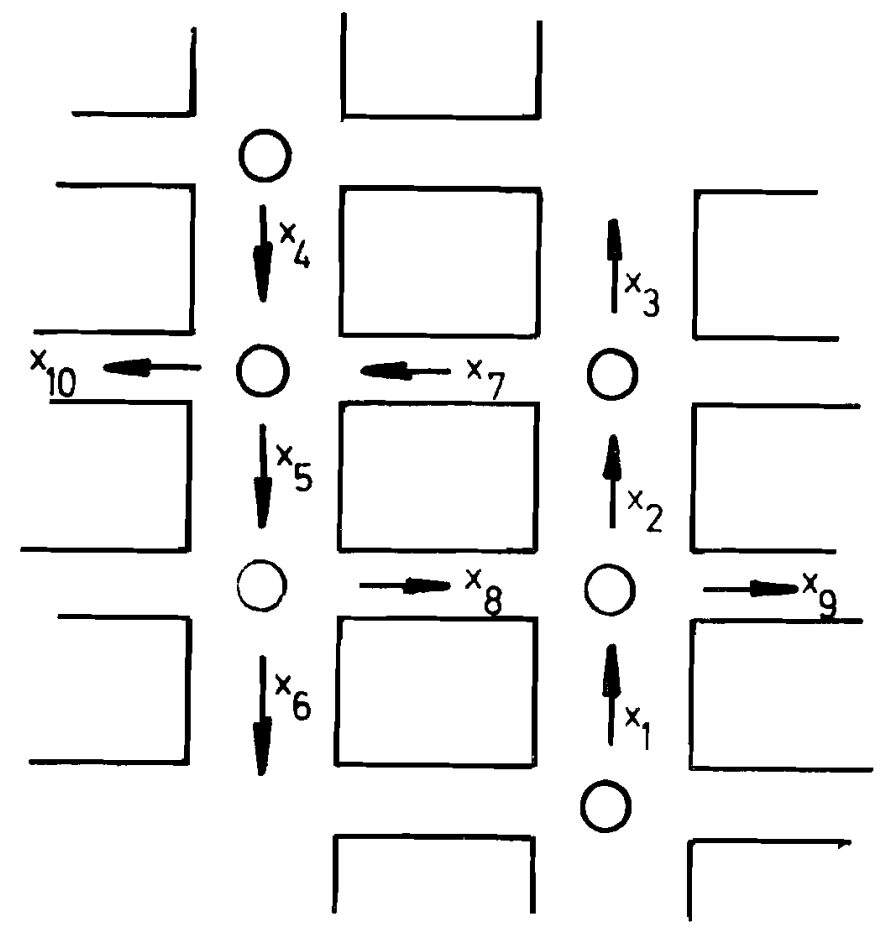

Figure 5. Urban Traffic Network

We assume that the network is over-saturated; i.e., at one or more intersections traffic demand exceeds capacity. Let $x_{i}(t)$ be the number of cars waiting at intersection $i$, and let $u_{i}(t)$ denote the number of cars leaving intersection $i$ during the green light. If we assume that the travel time between two intersections is small compared to the waiting time, then the dynamics of the process are reasonably well described by the equations

$$
x(t+1)=x(t)+G u(t)+q(t),
$$

where the vector $q(t)$ has components $q_{i}(t)$ representing the external traffic arriving at intersection $i$ during period $t$. It is clear from Figure 5 that the flows $u_{3}, u_{6}, u_{9}$, and $u_{10}$ are flows out of the network.

The control matrix $G$ takes the form 


$$
G=\left[\begin{array}{rrrrrrrrrr}
-1 & 0 & 0 & 0 & 0 & 0 & 0 & 0 & 0 & 0 \\
\mathrm{~s}_{1} & -1 & 0 & 0 & 0 & 0 & 0 & \mathrm{r}_{8} & 0 & 0 \\
0 & \mathrm{~s}_{2} & -1 & 0 & 0 & 0 & 0 & 0 & 0 & 0 \\
0 & 0 & 0 & -1 & 0 & 0 & 0 & 0 & 0 & 0 \\
0 & 0 & 0 & \mathrm{~s}_{4} & -1 & 0 & \mathrm{r}_{7} & 0 & 0 & 0 \\
0 & 0 & 0 & 0 & \mathrm{~s}_{5} & -1 & 0 & 0 & 0 & 0 \\
0 & \mathrm{r}_{2} & 0 & 0 & 0 & 0 & -1 & 0 & 0 & 0 \\
0 & 0 & 0 & 0 & \mathrm{r}_{5} & 0 & 0 & -1 & 0 & 0 \\
\mathrm{r}_{1} & 0 & 0 & 0 & 0 & 0 & 0 & \mathrm{~s}_{8} & -1 & 0 \\
0 & 0 & 0 & \mathrm{r}_{4} & 0 & 0 & \mathrm{~s}_{7} & 0 & 0 & -1
\end{array}\right]
$$

The elements $r_{i}$ and $s_{i}$ denote the percentage of cars turning right or left $\left(r_{i}\right)$ and going straight ahead $\left(s_{i}\right)$.

on psychological grounds, it is reasonable to impose the control constraints

$$
M_{i} \leq u_{i}(t) \leq u_{i}, \quad i=1, \ldots, 10,
$$

where $M_{i}$ and $U_{i}$ represent the minimal and maximal number of cars that are acceptable during a given green time.

The basic problem is now quite simple: given an initial state $x(0)$, assumed to be an oversaturated condition, is there a control policy $u(t)$ which transfers $x(t)$ to an undersaturated region within a prescribed time $T$ ?

\section{E. Energy Systems [21]}

As a final example, we look at a time-varying version of an energy supply-demand process surveyed in [21]. The model describes a typical input/output economy with energy considerations included by means of the system output.

Assume that $x_{i}(t)$ represents the total production up to time $t$ of economic sector $i$, while we let $u_{i}(t)$ denote the total demand up to time $t$ for the output of sector $i$. Further, we suppose that $a_{i j}$ is a constant representing the amount of 
production output of sector $i$ which is needed by sector $j$ to produce one unit of production, $i, j=1, \ldots, n$. It is fairly easy to see that the dynamics of this elementary model are

$$
x_{i}(t+1)=\sum_{j=1}^{n} a_{i j} x_{j}(t)+u_{i}(t) \quad, \quad i=1, \ldots, n \text {. }
$$

Now suppose that we introduce energy considerations into the picture. Let $\mathrm{E}_{i}$ represent the total energy output of sector $i$, and assume that a known fraction $e_{i k}$ of the total energy output of sector $i$ is sold to sector $k, i, k=1, \ldots, n$. Then

$$
E_{i}(t)=\sum_{k=1}^{n} e_{i k} x_{k}(t) \quad, \quad i=1, \ldots, n \text {. }
$$

The above problem is in standard form for reachability/ constructibility analyses. For instance, we could pose the important constructibility question as to whether, on the basis of past observed energy outputs, it is possible to uniquely determine the current total output $x_{i}(t)$ of each sector. Alternately, we could analyze the question of how to regulate consumer demands in order to stay within capacity constraints imposed upon the production process energy considerations.

It should by now be evident that the above model could be extended to incorporate time-varying coefficients $a_{i j}=a_{i j}(t)$, $e_{i k}=e_{i k}(t)$, nonlinear dynamics, and constraints on demands (positivity), and still be within the methodological bounds prescribed in this report. 


\section{$\underline{\text { References }}$}

1. McFadden, D. "On the Controllability of Decentralized Macroecoromic Systems: The Assignment Problem," in Math. Systems Theory and Economics-I, H. Kuhn, Ed., springer Lecture Notes in OR \& Math. Economics, Vol. 11, New York, 1969.

2. Kalman, R., P. Falb, and M. Arbib, Topics in Mathematical Systems Theory, McGraw-Hill Co., New York 1969 .

3. Kalman, R. Lectures on Controllability and observability, Proc. CIME Summer School, Edizioni Cremonese, Rome, 1969.

4. Kalman, R. "On the General Theory of Control Systems," Proc. 1st IFAC Congress, Moscow, 1960.

5. Gabasov, R., and F. Kirillova, The Qualitative Theory of Optimal Processes, Nauka, Moscow, 1969 (Russian).

6. Brockett, R. Finite-Dimensional Linear Systems, John Wiley, $\varepsilon$ Sons, $\overline{\text { New }}$ York, 1970 .

7. Markus, L. "Dynamic Keynesian Economic Systems: Control and Identification," in Math. System Theory and Economics-I, H. Kuhn, Ed., Springer Lecture Notes in OR \& Math. Economics, Vol. 11, New York, 1969.

8. Bruni, C., G. DiPillo, and G. Koch, "Bilinear Systems: An Appealing Class of 'Nearly Linear' Systems in Theory and Application," IEEE Tran. Auto. Cont. AC-19 (1974), 334-348.

9. Brockett, R. "Lie Algebras and Lie Groups in Control Theory," in Proc. NATO Advanced Study Institute on Geometric and Algebraic Methods for Nonlinear Systems, London, 1973 .

10. Isidori, A. "New Results on the Abstract Realization Theory of Nonlinear Input-Output Functions," Ricerche di Automatica $\underline{5}(1974), 52-61$.

11. Lobry, C. "Dynamical Polysystems and Control Theory," Proc. NATO Advanced Study Institute on Geometric and Algebraic Methods for Nonlinear Systems, London, 1973.

12. Walters, C. "Adaptive Control Problems in Renewable Resource Development," Internal Paper, IIASA, 1975.

13. Walters, C. "Optimal Harvest Strategies for Salmon in Relation to Environmental Variablity and Uncertainty About Production Parameters," Internal Paper, IIASA, 1975. 
14. Sz. Nagy, A. "State-Space Approach to Hydrology," Symposium on Math. Modelling in Hydrology, University College, Galway, Ireland, April 1974 .

15. Casti, J. "Algorithms for the stochastic Inflow-Nonlinear Objective water Reservoir Control Problem," IIASA Workshop on Vistula and Tisza River Basins, $\overline{\text { IIASA }}$ February 1975 .

16. Sz. Nagy, A. "On the Optimal Stochastic Control of water Resource Systems," Internal Paper, IIASA, 1975.

17. Mehra, R. "An Optimal Control Approach to National Settlement System Planning," IIASA RM-75-58, IIASA, 1975.

18. Strobel, H. "Transportation, Automation, and the Quality of Urban Living," IIASA RR-75-34, IIASA, 1975.

19. Kalman, R., B. Ho, and K. Narendra, "Controllability of Linear Dynamical Systems," Contr. to Diff. Eqs. 1 $(1963), 189-213$.

20. Wei, J., and E. Norman, "On the Global Representation of the Solutions of Linear Differential Equations as a Product of Exponentials," Proc. Amer. Math. Soc. $15(1964), 327-334$.

21. Charpentier, J.P. "Overview on Technigues and Models Used in the Energy Field," IIASA RM-75-8, IIASA, 1975. 